\title{
In memoriam Thierry Frébourg
}

\author{
Pascale Saugier-Veber, Mireille Cossée, Nicolas Lévy
}

Le Professeur Thierry Frébourg est décédé brutalement le 13 mars 2021 à l'âge de 60 ans. Ses collègues et amis généticiens de la Filière FILNEMUS lui rendent hommage, lui qui a tant fait pour les maladies rares.

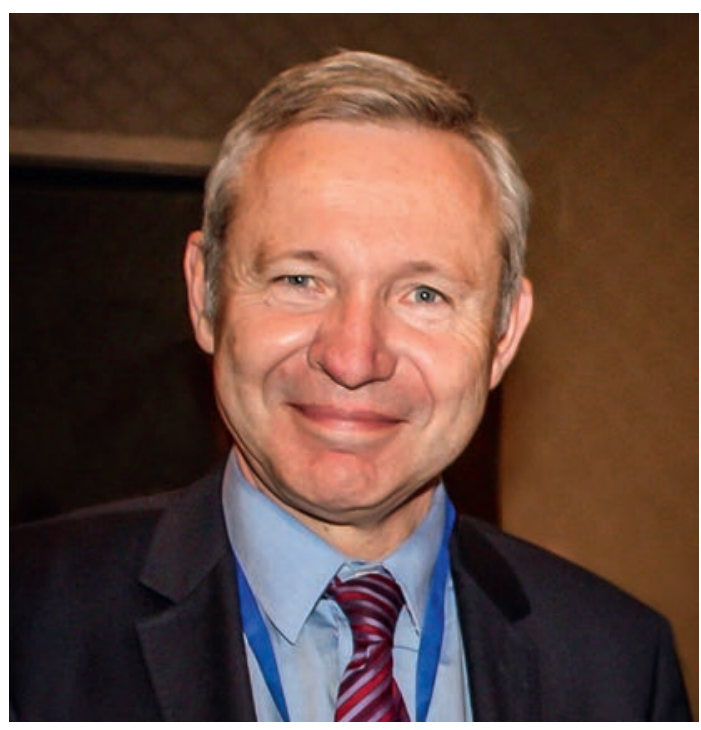

Toujours impeccablement soigné et d'une élégance à peine désuète mais si charmante, Thierry Frébourg était un homme d'une grande culture dont l'apparence pouvait cacher, pour ceux qui le connaissaient moins, l'aventurier qu'il était dans l'âme et par l'action. Aventurier, il fonda ex nihilo le service de génétique du CHU de Rouen au retour de son post-doctorat à la Harvard Medical School de Boston lors duquel il avait débuté ses travaux sur le syndrome de Li-Fraumeni. Parallèlement, il créa un petit groupe de recherche qui deviendra l'unité Inserm U1245, consacrée à la génomique et à la médecine personnalisée dans le cancer et les affections neurologiques. Au fil des années, en brillant chef d'orchestre, Thierry Frébourg sut fédérer autour de lui les acteurs de génétique clinique, de génétique moléculaire et de cytogénétique en un département de génétique adossé à cette unité Inserm, et créa le Rouen Institute for Research and Innovation in Biomedicine dont il fut ensuite le directeur. Brillant, curieux, enthousiaste, doté d'une force de travail impressionnante, il impulsait une véritable dynamique dans la communauté médicale et scientifique normande. Architecte, il a largement contribué à façonner la génétique française.
Républicain, il a porté haut les valeurs de probité, de service public, d'équité d'accès aux soins et d'engagement au service du collectif. Humaniste dans l'âme, il s'est aussi attaché à défendre une conception de l'éthique dans l'exercice de la génétique conforme à celle du Pr Jean-François Mattéi. Sans omettre ses actions pour le développement de la génétique dans sa Normandie natale avec la labellisation d'une Fédération Hospitalo-Universitaire (FHU), ni oublier ses contributions scientifiques majeures, tout particulièrement dans le syndrome de Li-Fraumeni et la génétique des cancers, nous nous attacherons à montrer la façon dont il a œuvré pour la structuration de la génomique en France.

Thierry Frébourg fut projeté sur la scène nationale lors de sa nomination dans la commission Marescaux. Mise en place en décembre 2008 par le Président de la République de l'époque, M. Nicolas Sarkozy, et dirigée par le Professeur Jacques Marescaux, cette commission avait comme mission de se pencher sur l'avenir des CHU et d'être force de propositions. La commission remit son rapport en mai 2009 et permit notamment la création de six instituts hospitalo-universitaires (IHU) dès juillet 2010 : Imagine, Méditerranée Infection, Institut de chirurgie hybride mini-invasive guidée par l'image (Mix Surg), Institut du Cerveau et de la Moelle épinière (ICM), Institut de Cardiométabolique et Nutrition (ICAN) et Institut de rythmologie et de modélisation cardiaque (Liryc).

L'acuité intellectuelle exceptionnelle de Thierry Frébourg, associée à un esprit de synthèse hors du commun, furent légitimement reconnus puisqu'il fut alors appelé à co-diriger de 2013 à 2016 l'Institut Thématique Multi-Organisme (ITMO) de Génétique, Génomique et Bioinformatique (GGB) d'AVIESAN (Alliance pour les Sciences de la Vie et de la Santé) aux côtés de Thierry Grange. Leurs travaux définirent comme priorité d'intégrer et d'anticiper les implications du développement du séquençage à haut débit dans la recherche en biologie et en santé, non seulement en génétique humaine mais également en génétique microbienne et animale. L'actualité Covid met précisément en lumière l'importance 
de la génomique dans le suivi épidémique des souches virales de SARS-CoV-2. La bioinformatique et les sciences de la modélisation ont une place majeure pour le déploiement du séquençage à haut débit comme des autres analyses omiques telles la transcriptomique, la protéomique, la métabolomique, ou l'interactomique. Dans leur rapport rendu le 17 mai 2013, Thierry Frébourg et Thierry Grange faisaient le bilan des forces et faiblesses de la France en matière de génomique. Ils concluaient à l'importance de déployer des plateformes de séquençage à très haut débit, rapidement opérationnelles, capables d'assurer des analyses massives correspondant aux besoins en exomes et génomes, avec un financement public initial pour permettre leur auto-financement ultérieur. Dans ce rapport, véritable préfigurateur du futur plan France Médecine Génomique 2025, on reconnaîtra également l'attachement de Thierry Frébourg à la nécessaire réflexion éthique associée à la médecine génomique, incitant à définir un cadre éthique et réglementaire indispensable pour l'information des personnes, l'utilisation des données dans le contexte du diagnostic et de la recherche et allant jusqu'au partage de ces données. Ainsi, c'est au titre de co-directeur de l'ITMO Génétique, Génomique et Bioinformatique que Thierry Frébourg débuta l'aventure du Plan France Médecine Génomique. Dès 2012, des initiatives majeures ont émergé au niveau international avec le "100,000 Genomes Project " au Royaume-Uni en 2012, lancé par le Premier Ministre David Cameron, et le projet "Precision Medicine Initiative " aux États-Unis en 2014, sous l'impulsion du Président Obama. Thierry Frébourg, toujours à la pointe des dernières avancées technologiques et scientifiques, a largement commenté ces initiatives au sein de la communauté médicale et scientifique, en vue de leur déclinaison au niveau national. En effet, à l'époque où la Fondation Maladies Rares, dès 2012, propose les premiers financements de séquençage d'exomes et de génomes entiers via ses appels à projets, Thierry Frébourg est chargé de représenter l'Inserm au conseil d'administration de la Fondation. Durant les quatre années où il assure cette mission, il déploiera une énergie sans retenue, afin de convaincre les représentants des $\mathrm{CHU}$, des Universités et des associations de malades, non seulement du bien-fondé de ces programmes mais aussi de la nécessité de combler notre retard au bénéfice de la connaissance et des malades. Défenseur farouche de la médecine de précision, tout en restant attaché à ses valeurs de généticien clinicien, il défendra la génétique en tant que discipline médicale, intégrant la prise en charge, le diagnostic moléculaire et la recherche dans un continuum permanent. Cette période d'intenses réflexions sur les contours de la médecine de demain se concrétisera à l'occasion de deux événements majeurs et parallèles. En cette année 2015, la thématique des maladies rares est retenue dans le cadre des 6èmes Rencontres Internationales de la Recherche (RIR) au Collège de France à Paris. Ces rencontres sont parrainées par le Président de la République et portées conjointement par ARIIS (l'Alliance pour la Recherche et l'Innovation des Industries de Santé) et AVIESAN. Pour AVIESAN, c'est Thierry Frébourg qui sera le grand organisateur et définira le thème " des maladies rares à la médecine personnalisée ". Se déroulant en présence de Mme Marisol Touraine, ministre des Affaires sociales et de la Santé et de M. Thierry Mandon, secrétaire d'État chargé de l'Enseignement supérieur et de la Recherche, l'objectif était de présenter l'excellence de la recherche française dans le domaine et de stimuler les partenariats entre recherche académique et recherche industrielle dans le champ des maladies rares. Thierry Frébourg connaissait l'enjeu et l'importance d'atteindre cet objectif car c'est dans ce contexte stratégique que le 17 avril 2015, le Premier Ministre, M. Manuel Valls, adressa une lettre de mission au Président d'AVIESAN, le Professeur Yves Lévy afin d'introduire la médecine génomique dans le parcours de soins. Thierry Frébourg fut naturellement appelé à appartenir au comité de pilotage de ce plan composé de 35 membres éminents autour du Professeur Yves Lévy. Quatre groupes de travail furent constitués qui eurent pour objectif d'analyser la situation nationale et internationale, de définir les enjeux industriels et de proposer des infrastructures et des projets pilotes. Thierry Frébourg pilota le groupe de travail "État des lieux en France : recherche et applications techniques " et, avec Jean-François Deleuze, le groupe de travail transversal "Innovation: projets pilotes". Une phase de concertation entre octobre 2015 et mars 2016 mobilisera plus de 160 professionnels de tous horizons : professionnels de santé, administration centrale, institutionnels (CNAM, HAS, CGI, École d'économie de Toulouse), industriels et associations de patients. Le rapport du Plan France Médecine Génomique 2025 (PFMG 2025) fut remis au Premier Ministre le 22 juin 2016. L'ambition était bien que la France se dote d'une filière médicale et industrielle autour de la génomique. Grâce à la génomique, la France, à l'instar d'autres puissances mondiales, entrerait dans l'ère de la médecine personnalisée afin d'améliorer le diagnostic, de préciser le pronostic sur des bases moléculaires et de développer la théranostique (contraction de thérapie et diagnostic) dans le parcours de soins tout d'abord 
des maladies rares, puis du cancer et des maladies communes. Le plan prévoyait également une forte valence de recherche et d'innovation: il s'agissait d'intégrer les avancées scientifiques dans le soin afin de faciliter l'accès à l'innovation sur tout le territoire français, notamment par l'intermédiaire du Collecteur Analyseur de Données (CAD), l'organe de centralisation de toutes les variations génomiques interprétées. Enfin, sur le plan industriel, le PFMG 2025 devait permettre le développement du secteur du numérique autour du traitement de données massives, du secteur informatique et bioinformatique, et du secteur biomédical. Le PFMG2025 définissait 3 objectifs. Le premier visait à placer la France dans le peloton de tête des grands pays engagés dans la médecine personnalisée, le second était de préparer l'intégration de la médecine de précision dans le parcours de soins tout d'abord des maladies rares, puis du cancer et des maladies communes et le troisième était de faire émerger une filière de médecine génomique capable d'être un levier d'innovation scientifique et technologique, de valorisation industrielle et de croissance économique. Le plan définissait l'infrastructure nécessaire à la prise en charge des besoins diagnostiques génomiques de la population française dans les domaines des maladies rares, du cancer et des maladies communes, avec une montée en charge jusqu'à l'horizon 2025 . Il précisait ce que doivent être les plateformes de séquençage très haut-débit, estimant leur nombre nécessaire ainsi que leurs modalités prévisionnelles de fonctionnement. Enfin, ce plan prévoyait la mise en place du $\mathrm{CAD}$ ainsi qu'un centre de référence, d'innovation, d'expertise et de transfert (CREFIX) chargé du choix des technologies et de la bioinformatique. Pour évaluer l'efficacité de ces infrastructures aussi bien que pour définir le parcours de soins et évaluer les coûts, quatre projets pilotes furent conçus : POPGEN pour évaluer la variabilité génétique dans la population française, DEFIDIAG autour de la déficience intellectuelle pour évaluer l'utilité de la génomique dans les maladies rares, GLUCOGEN autour du diabète pour évaluer l'utilité de la génomique dans les maladies communes et MULTIPLI pour évaluer l'utilité de la génomique dans le cancer. On connait la suite : les plateformes SeqOIA (Île-de-France) et AURAGEN (Auvergne-Rhône-Alpes) ont été sélectionnées le 17 juillet 2017 parmi 12 projets soumis. Ironie de l'histoire, les projets pilotes ont démarré après le déploiement du plan. Pourtant, dès novembre 2016, Thierry Frébourg avec Hélène Dollfus à ses côtés, avait pris en charge la coordination du projet pilote DEFIDIAG. Porté par AVIESAN, ce projet devait répondre à des questions scientifiques et médicales concernant la stratégie d'analyse mais également permettre une évaluation économique afin de répondre à la question de la prise en charge financière par la nation. L'avis favorable du CPP puis de la CNIL, ont été obtenus respectivement en juin 2019 et novembre 2020. La crise sanitaire du COVID-19 a retardé les premières inclusions débutées en mars 2020. À ce jour, près de 850 trios ont été inclus et les premiers résultats ont été rendus. Thierry Frébourg aurait mérité de voir les conclusions de ce projet pour lequel il a ardemment ouvré.

Thierry Frébourg ancrait cet engagement au service du collectif dans ses valeurs humanistes. Il s'est toujours attaché à défendre une éthique de l'accompagnement assez opposée à l'attitude américaine. Il osait porter le verbe haut pour dénoncer ce qu'il percevait comme des dérives, notamment dans les applications de la génétique pour le dépistage en population générale. S'engager en éthique était juste une exigence morale.

Chacun d'entre nous se souvient de ses brillantes présentations, de ses talents d'orateur, de son esprit de synthèse, de son enthousiasme et de sa verve, signes d'une immense générosité qui nous emportaient avec lui. Quelle énergie il aura déployée pour structurer, innover, construire, rassembler avec comme maître mot : défendre l'excellence. Thierry était le plus parfait représentant du monde hospitalier, universitaire et de recherche. Un talent et une énergie folle pour fédérer et construire, mener des équipes, tout en restant un grand médecin, un magnifique enseignant reconnu comme tel par ses étudiants et un brillant scientifique. Ses nombreux travaux majeurs dans le syndrome de Li-Fraumeni, de la génétique des cancers et des maladies neuropsychiatriques lui survivront. Thierry Frébourg était naturellement attendu pour s'impliquer dans d'autres projets d'envergure nationale et internationale. Son décès prématuré nous prive de son esprit visionnaire, de sa force de travail redoutable, de son art de fédérer des talents autour de lui. Puissent sa force de vie incroyable, son sempiternel sourire, sa générosité et son incorrigible optimisme nous porter pour poursuivre son action au service de la génétique française à laquelle il manque déjà tellement.

\section{In memoriam Thierry Frébourg}

\section{LIENS D'INTÉRÊT}

Les auteurs déclarent n'avoir aucun lien d'intérêt concernant les données publiées dans cet article. 